\title{
Density of Eigenvalues of Random Normal Matrices with an Arbitrary Potential, and of Generalized Normal Matrices ${ }^{\star}$
}

\author{
Pavel ETINGOF* and Xiaoguang MA \\ Department of Mathematics, Massachusetts Institute of Technology, \\ 77 Massachusetts Ave., Cambridge, MA 02139 USA \\ E-mail: etingof@math.mit.edu,xma@math.mit.edu \\ * URL: http://www-math.mit.edu/ etingof/
}

Received December 05, 2006, in final form March 03, 2007; Published online March 14, 2007

Original article is available at http://www.emis.de/journals/SIGMA/2007/048/

\begin{abstract}
Following the works by Wiegmann-Zabrodin, Elbau-Felder, Hedenmalm-Makarov, and others, we consider the normal matrix model with an arbitrary potential function, and explain how the problem of finding the support domain for the asymptotic eigenvalue density of such matrices (when the size of the matrices goes to infinity) is related to the problem of Hele-Shaw flows on curved surfaces, considered by Entov and the first author in 1990-s. In the case when the potential function is the sum of a rotationally invariant function and the real part of a polynomial of the complex coordinate, we use this relation and the conformal mapping method developed by Entov and the first author to find the shape of the support domain explicitly (up to finitely many undetermined parameters, which are to be found from a finite system of equations). In the case when the rotationally invariant function is $\beta|z|^{2}$, this is done by Wiegmann-Zabrodin and Elbau-Felder. We apply our results to the generalized normal matrix model, which deals with random block matrices that give rise to $*$-representations of the deformed preprojective algebra of the affine quiver of type $\hat{A}_{m-1}$. We show that this model is equivalent to the usual normal matrix model in the large $N$ limit. Thus the conformal mapping method can be applied to find explicitly the support domain for the generalized normal matrix model.
\end{abstract}

Key words: Hele-Shaw flow; equilibrium measure; random normal matrices

2000 Mathematics Subject Classification: 15A52

\section{Introduction}

The normal matrix model became a focus of attention for many mathematical physicists after the recent discovery (see e.g. $[11,6,7,8]$ ) of its unexpected connections to the 2-dimensional dispersionless Toda hierarchy and the Laplacian growth model (which is an exactly solvable model describing free boundary fluid flows in a Hele-Shaw cell or porous medium). The original normal matrix model contained a potential function whose Laplacian is a positive constant, but later in [12], Wiegmann and Zabrodin considered a more general model, where the potential function was arbitrary. This is the model we will consider in this paper.

In the normal matrix model with an arbitrary potential function, one considers the random normal matrices of some size $N$ with spectrum restricted to a compact domain $D^{1}$ and probability measure

$$
P_{N}(M) \mathrm{d} M=Z_{N}^{-1} \exp (-N \operatorname{tr} W(M)) \mathrm{d} M
$$

${ }^{\star}$ This paper is a contribution to the Vadim Kuznetsov Memorial Issue 'Integrable Systems and Related Topics'. The full collection is available at http://www.emis.de/journals/SIGMA/kuznetsov.html

${ }^{1}$ The compactness of $D$ is needed to guarantee convergence of the arising integrals. 
where $\mathrm{d} M$ is the measure on the space of normal matrices induced by the Euclidean metric on all complex matrices, $W$ is a potential function (a real function on $\mathbb{C}$ with some regularity properties, e.g. continuous), and $Z_{N}$ is a normalizing factor.

In the original works on the normal matrix model, the potential was

$$
W(z)=\beta|z|^{2}-P(z)-\overline{P(z)},
$$

where $P$ is a complex polynomial of some degree $d$, and $\beta$ a positive real number. For this type of potential, it was shown in the works $[11,6,7,8]$ (and then proved rigorously in [3]) that under some conditions on the potential, the asymptotic density of eigenvalues is uniform with support in the interior domain of a closed smooth curve. This curve is a solution of an inverse moment problem, appearing in the theory of Hele-Shaw flows with a free boundary. Thus, applying the conformal mapping method (see [10] and references therein), one discovers that the conformal map of the unit disk onto the outside of this curve which maps 0 to $\infty$ is a Laurent polynomial of degree $d$. This allows one to find the curve explicitly up to finitely many parameters, which can be found from a finite system of algebraic equations.

In [12], Wiegmann and Zabrodin generalized this analysis to an arbitrary potential function. They showed that the density of eigenvalues is the Laplacian of the potential function, and the eigenvalues are concentrated in the domain which can be determined from an appropriate inverse moment problem. This was proved rigorously in the paper [5], which extends the Elbau-Felder work to the case of an arbitrary potential.

One of the goals of the present paper is to use the generalized conformal mapping method, developed in [4] by Entov and the first author for studying Hele-Shaw flows with moving boundary for curved surfaces, to calculate the boundary of the region of eigenvalues explicitly in the case when

$$
W(z)=\Phi\left(|z|^{2}\right)-P(z)-\overline{P(z)}
$$

where $\Phi$ is a function of one variable. In this case, the conformal map of the disk onto the outside of the curve is no longer algebraic, but one can still give an explicit answer in terms of a contour integral.

Another goal is to extend the above results to the case of generalized normal matrix model. In this model, we consider block complex matrices of a certain kind with commutatation relations similar to the definition of a normal matrix; they give rise to $*$-representations of the deformed preprojective algebra of the affine quiver of type $\hat{A}_{m-1}$. We prove that the problem of computing the asymptotic eigenvalue distribution for this model, as the size of the matrices goes to infinity, is equivalent to the same problem for the usual normal matrix model. This allows one to find the boundary of the eigenvalue region explicitly if the potential is given by (1).

The structure of this paper is as follows. In Section 2, we state some basic facts about the normal matrix model. In Section 3, we define the generalized normal matrix model, and write down the probability measure in this model. In Section 4, we recall some facts about the equilibrium measure and explain that the asymptotic eigenvalue distribution tends to the equilibrium measure in the normal matrix model and the generalized normal matrix model. In

Section 5, we use the singular point method from $[4,10]$ to reconstruct the boundary of the support domain of the equilibrium measure.

\section{The normal matrix model with an arbitrary potential function}

Let $D$ be a domain in the complex plane $\mathbb{C}$. We consider the set

$$
\mathcal{N}(D)=\left\{M \in \operatorname{Mat}_{N}(\mathbb{C}) \mid\left[M, M^{\dagger}\right]=0, \operatorname{spectrum}(M) \subset D\right\}
$$


of normal matrices with spectrum in the domain $D$. Let $\mathrm{d} M$ be the measure on $\mathcal{N}(D)$ induced by the Euclidean metric on $\operatorname{Mat}_{N}(\mathbb{C})$. It is well known (see e.g. $[9,1]$ ), that in terms of the eigenvalues this measure on $\mathcal{N}(\mathbb{C})$ is given by the formula

$$
\mathrm{d} M=\prod_{1 \leq i<j \leq N}\left|z_{i}-z_{j}\right|^{2} \prod_{i=1}^{N} \mathrm{~d}^{2} z_{i} \mathrm{~d} U
$$

where $M=U \operatorname{diag}\left(z_{1}, \ldots, z_{N}\right) U^{\dagger}, U \in U(N)$, and $\mathrm{d} U$ denotes the normalized $U(N)$-invariant measure on the flag manifold $U(N) / U(1)^{N}$.

Now let $W: \mathbb{C} \rightarrow \mathbb{R}$ be a continuous function. If $M$ is a normal matrix, then we can define $W(M)$ to be $\operatorname{diag}\left(W\left(z_{1}\right), \ldots, W\left(z_{N}\right)\right)$ in an orthonormal basis in which $M=\operatorname{diag}\left(z_{1}, \ldots, z_{N}\right)$. It follows from the above that the probability measure on $\mathcal{N}(D)$ with potential function $W$ is given by

$$
P_{N}(M) \mathrm{d} M=Z_{N}^{-1} e^{-N \sum_{i} W\left(z_{i}\right)} \prod_{1 \leq i<j \leq N}\left|z_{i}-z_{j}\right|^{2} \prod_{i=1}^{N} \mathrm{~d}^{2} z_{i} \mathrm{~d} U,
$$

where $Z_{N}=\int_{D^{N}} e^{-N \sum_{i} W\left(z_{i}\right)} \prod_{1 \leq i<j \leq N}\left|z_{i}-z_{j}\right|^{2} \prod_{i=1}^{N} \mathrm{~d}^{2} z_{i}$. Here we assume that the integral is convergent (this is the case, for instance, if $D$ is compact).

\section{The generalized normal matrix model}

\subsection{Generalized normal matrices}

Let us consider the following generalization of normal matrices. Let $m \geq 1$ be an integer. For a fixed collection $\lambda=\left(\lambda_{1}, \ldots, \lambda_{m}\right)$ of real numbers such that $\sum_{i} \lambda_{i}=0$, and a domain $D$, we define $\mathcal{N}_{m}(\lambda, D)$ to be the subset of $A \in \operatorname{Mat}_{m N}(\mathbb{C})$ satisfying the following conditions:

for any $A \in \mathcal{N}_{m}(\lambda, D)$,

- If $A_{i j}, i, j=1, \ldots, m$ are $N \times N$ blocks of $A$, then $A_{i j}=0$ unless $j-i=1 \bmod m$;

- The spectrum of $A_{12} A_{23} \cdots A_{m 1}$ is contained in $D$;

- $\left[A, A^{\dagger}\right]=\left(\begin{array}{ccc}\lambda_{1} I_{N} & & \\ & \ddots & \\ & & \lambda_{m} I_{N}\end{array}\right)$, where $I_{N}$ is the identity matrix of size $N$.

Note that $\mathcal{N}_{1}(0, D)=\mathcal{N}(D)$, thus elements of $\mathcal{N}_{m}(\lambda, D)$ are a generalization of normal matrices. We will thus call them generalized normal matrices.

Remark 1. Generalized normal matrices are related in the following way to quiver representations. Let $Q$ be the cyclic quiver of type $\hat{A}_{m-1}$, and $\bar{Q}$ its double. Let $\Pi_{Q}(\lambda)$ be the deformed preprojective algebra of $Q$ with parameters $\lambda$ (see [2]). By definition, this algebra is the quotient of the path algebra of $\bar{Q}$ by the relation $\sum_{a \in Q}\left[a, a^{*}\right]=\sum \lambda_{i} e_{i}$, where $e_{i}$ are the vertex idempotents. The algebra $\Pi_{Q}$ has a $*$-structure, preserving $e_{i}$ and sending $a$ to $a^{*}$ and $a^{*}$ to $a$. It is easy to see that $\mathcal{N}_{m}(\lambda, D)$ is the set of all matrix *-representations of $\Pi_{Q}$ of dimension $N \delta$ (where $\delta=(1,1, \ldots, 1)$ is the basic imaginary root) such that the spectrum of the monodromy operator $a_{1} \cdots a_{m}$ is in $D$. 
Denote $A_{i, i+1}$ by $A_{i}$. The group $U(N)^{m}=\underbrace{U(N) \times U(N) \times \cdots \times U(N)}_{m \text { times }}$ acts naturally on $\mathcal{N}_{m}(\lambda, \mathbb{C})$ by the formula

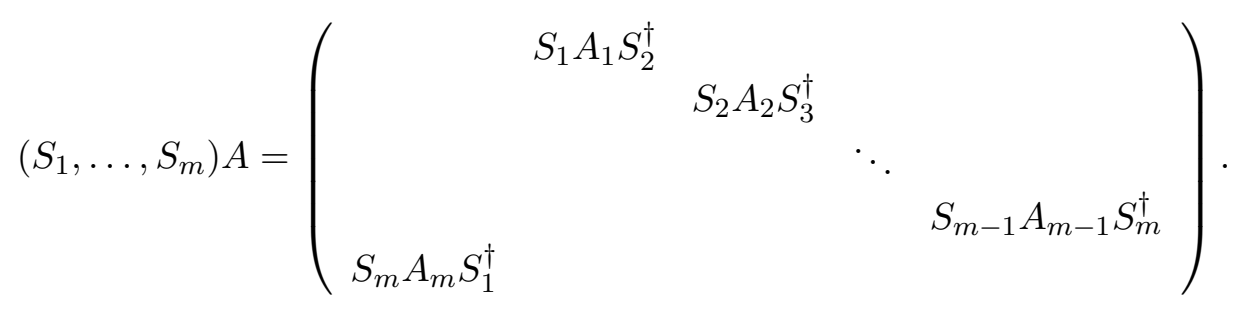

We have the following lemma, which is a generalization of the fact that a normal matrix diagonalizes in an orthonormal basis:

Lemma 1. For any element $A \in \mathcal{N}_{m}(\lambda, D)$, we can find an element $\left(S_{1}, \ldots, S_{m}\right) \in U(N)^{m}$ such that

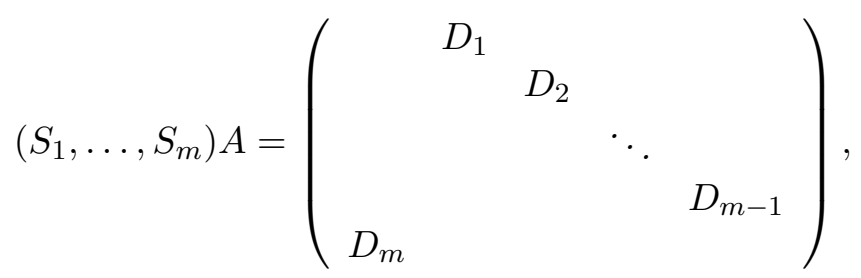

where $D_{i}$ are diagonal matrices.

Proof. From the definition, we have $A_{i} A_{i}^{\dagger}-A_{i-1}^{\dagger} A_{i-1}=\lambda_{i} I_{N}$, where the index is considered modulo $m$, and $\sum_{i=1}^{m} \lambda_{i}=0$.

Now consider a collection of $N$-dimensional unitary spaces $\left\{V_{i}\right\}_{i=1}^{m}$, and let us regard $A_{i}$ as a linear map $A_{i}: V_{i+1} \rightarrow V_{i}$. So $A_{i} A_{i}^{\dagger}$ is a hermitian endomorphism of $V_{i}$.

Now suppose that $v$ is an eigenvector of $A_{i} A_{i}^{\dagger}$ with eigenvalue $\nu$. We claim that then $A_{i-1} v$ (if it is nonzero) is an eigenvector of $A_{i-1} A_{i-1}^{\dagger}$ with eigenvalue $\nu-\lambda_{i}$. Indeed,

$$
A_{i-1} A_{i-1}^{\dagger} A_{i-1} v=A_{i-1}\left(A_{i} A_{i}^{\dagger}-\lambda_{i}\right) v=\left(\nu-\lambda_{i}\right) A_{i-1} v .
$$

Thus, denoting by $V_{i}(\nu)$ the eigenspace of $A_{i} A_{i}^{\dagger}$ with eigenvalue $\nu$, we find that $A_{i-1}: V_{i}(\nu) \rightarrow$ $V_{i-1}\left(\nu-\lambda_{i}\right)$. Since $V_{i}=\oplus_{\nu \in \mathbb{R}} V_{i}(\nu)$ (as $A_{i} A_{i}^{\dagger}$ is hermitian), it suffices to prove the lemma in the case when $A_{i} A_{i}^{\dagger}$ is a scalar in $V_{i}$, in which case the statement is easy.

\subsection{The Euclidean measure on generalized normal matrices}

First, let us consider the $N=1$ case. Pick real numbers $\alpha_{i}$ such that $\lambda_{i}=\alpha_{i}-\alpha_{i-1}$, and let $Q(x)=\prod_{i=1}^{m}\left(x+\alpha_{i}\right)$. A matrix

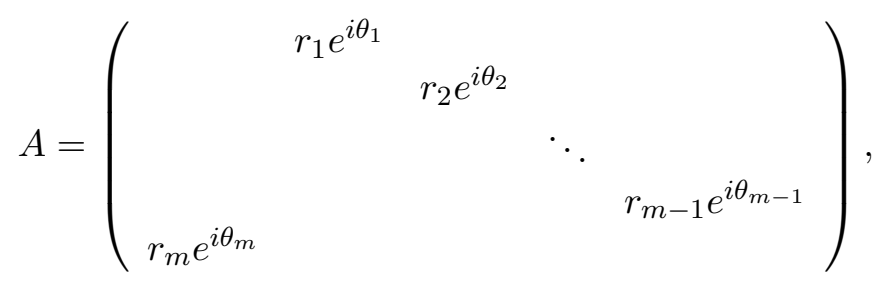

(where $r_{j} \geq 0, \theta_{j} \in[0,2 \pi)$ ) is in $\mathcal{N}_{m}(\lambda, \mathbb{C})$ if and only if

$$
r_{1}^{2}-r_{m}^{2}=\lambda_{1}=\alpha_{1}-\alpha_{m},
$$




$$
\begin{gathered}
r_{2}^{2}-r_{1}^{2}=\lambda_{2}=\alpha_{2}-\alpha_{1}, \\
\vdots \\
r_{m}^{2}-r_{m-1}^{2}=\lambda_{m}=\alpha_{m}-\alpha_{m-1} .
\end{gathered}
$$

Thus to each $A \in \mathcal{N}_{m}(\lambda, \mathbb{C})$ we can attach a real number $x=r_{i}^{2}-\alpha_{i}$, which is independent of $i$, and a complex number $z=\prod_{j=1}^{m} r_{j} e^{i \theta_{j}}$. It is easy to see that the point $(z, x)$ belongs to the surface

$$
\Sigma=\left\{(z, x) \in \mathbb{C} \times \mathbb{R} \mid x+\alpha_{i} \geq 0 \forall i, z \bar{z}=\prod_{i=1}^{m}\left(x+\alpha_{i}\right)\right\} .
$$

Moreover, it is clear that any point of $\Sigma$ corresponds to some $A$, and two matrices $A, A^{\prime}$ giving rise to the same point $(z, x)$ are conjugate. This implies that we have a bijection between the equivalence classes in $\mathcal{N}_{m}(\lambda, \mathbb{C})$ under the action of $U(1)^{m}$ and points of $\Sigma$. Writing $z=r e^{i \theta}$, we see that $x, \theta$ are coordinates on $\Sigma$, so we may write the Euclidean measure on $\mathcal{N}_{m}(\lambda, \mathbb{C})$ using the coordinates $x, \theta$.

Theorem 1. The Euclidean measure on $\mathcal{N}_{m}(\lambda, \mathbb{C})$ for $N=1$ is:

$$
\mu=\frac{1}{2} \sqrt{Q^{\prime}(x)} \mathrm{d} x \mathrm{~d} \theta \mathrm{d} U,
$$

where $\mathrm{d} U$ is the Haar measure on $U(1)^{m} / U(1)$.

Proof. We have $r_{i}=\sqrt{x+\alpha_{i}}$. Thus the parametrized real curve $\left\{\left(r_{1}(x), \ldots, r_{m}(x)\right) \mid x \in \mathbb{R}\right\}$ has length element

$$
\mathrm{d} s=\sqrt{\sum_{i} r_{i}^{\prime}(x)^{2}} \mathrm{~d} x=\frac{1}{2} \sqrt{\sum_{i} \frac{1}{x+\alpha_{i}}} \mathrm{~d} x .
$$

This implies that the Euclidean measure on $\mathcal{N}_{m}(\lambda, \mathbb{C})$ is

$$
\begin{aligned}
\mathrm{d} \mu & =\frac{1}{2} \sqrt{\sum_{i} \frac{1}{x+\alpha_{i}}} \mathrm{~d} x \prod_{j} r_{j} \mathrm{~d} \theta_{j}=\frac{1}{2} \sqrt{\sum_{i} \prod_{j \neq i}\left(x+\alpha_{j}\right)} \mathrm{d} x \mathrm{~d} \theta_{1} \cdots \mathrm{d} \theta_{m} \\
& =\frac{1}{2} \sqrt{Q^{\prime}(x)} \mathrm{d} x \mathrm{~d} \theta_{1} \cdots \mathrm{d} \theta_{m}=\frac{1}{2} \sqrt{Q^{\prime}(x)} \mathrm{d} x \mathrm{~d} \theta \mathrm{d} U,
\end{aligned}
$$

as desired.

Let us now consider the case of general $N$. From Lemma 1, we know that under the action of $U(N)^{m}$, the equivalence class of $A \in \mathcal{N}_{m}(\lambda, \mathbb{C})$ can be represented by $m$ diagonal matrices

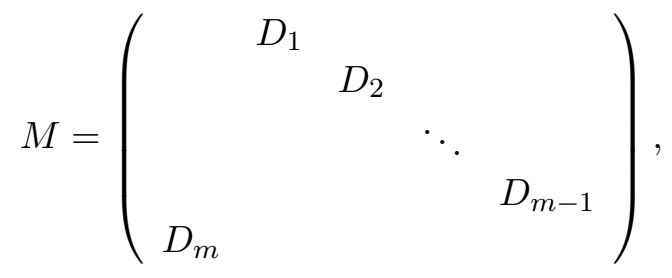

where $D_{i}=\operatorname{diag}\left(z_{1}^{i}, \ldots, z_{N}^{i}\right)$.

From the definition

$$
D_{1} D_{1}^{\dagger}-D_{m}^{\dagger} D_{m}=\lambda_{1} I_{N}
$$




$$
\begin{aligned}
& D_{2} D_{2}^{\dagger}-D_{1}^{\dagger} D_{1}=\lambda_{2} I_{N}, \\
& \quad \vdots \\
& D_{m} D_{m}^{\dagger}-D_{m-1}^{\dagger} D_{m-1}=\lambda_{m} I_{N} .
\end{aligned}
$$

So we have $z_{j}^{i} \overline{z_{j}^{i}}-z_{j}^{i+1} \overline{z_{j}^{i+1}}=\alpha_{i}-\alpha_{i+1}$. Let $x_{j}=z_{j}^{i} \overline{z_{j}^{i}}-\alpha_{i}$ and $z_{j}=\prod_{i} z_{j}^{i}$, then we have

$$
\left|z_{j}\right|^{2}=\prod_{i}\left(x_{j}+\alpha_{i}\right), \quad j=1, \ldots, N .
$$

Thus $\left(\left(z_{1}, x_{1}\right), \ldots,\left(z_{N}, x_{N}\right)\right)$ is a point on $\Sigma^{N} / S_{N}$.

Similarly to $N=1$ case, it is easy to show that this gives rise to a bijection between conjugacy classes of elements of $\mathcal{N}_{m}(\lambda, \mathbb{C})$ and points of $\Sigma^{N} / S_{N}$. Using this fact and combining the method of computation for usual normal matrices with the $N=1$ case, one gets the following result.

Theorem 2. The Euclidean measure on $\mathcal{N}_{m}(\lambda, \mathbb{C})$ has the form

$$
\mathrm{d} M=\frac{1}{2^{N}} \prod_{i} \sqrt{Q^{\prime}\left(x_{i}\right)} \prod_{i<j}\left|z_{i}-z_{j}\right|^{2} \mathrm{~d} x_{1} \cdots \mathrm{d} x_{N} \mathrm{~d} \theta_{1} \cdots \mathrm{d} \theta_{N} \mathrm{~d} U,
$$

where $\mathrm{d} U$ is the normalized invariant measure on $U(N)^{m} / U(1)^{N}$.

Proof. At first, consider the subset $\mathcal{N}_{m}^{\text {diag }}(\lambda, \mathbb{C})$ of $\mathcal{N}_{m}(\lambda, \mathbb{C})$ consisting of the elements $M$ of the form (3). Then by Theorem 1, the measure on $\mathcal{N}_{m}^{\text {diag }}(\lambda, \mathbb{C})$ induced by the Euclidean metric is the product measure:

$$
\mu_{\text {diag }}=\frac{1}{2^{N}} \prod_{i} \sqrt{Q^{\prime}\left(x_{i}\right)} \mathrm{d} x_{1} \cdots \mathrm{d} x_{N} \mathrm{~d} \theta_{1} \cdots \mathrm{d} \theta_{N} \mathrm{~d} U_{\text {diag }},
$$

where $\mathrm{d} U_{\text {diag }}$ is the Haar measure on $U(1)^{N m} / U(1)^{N}$.

Now consider the contribution of the off-diagonal part. Consider the elements

$$
\left\{v_{i, j}=E_{i, j}-E_{j, i}, w_{i, j}=\sqrt{-1}\left(E_{i, j}+E_{j, i}\right) \mid 0 \leqslant i<j \leqslant N\right\}
$$

of the Lie algebra of $U(N)$.

Let $V_{i, j, k}, W_{i, j, k}$ be the derivatives of $\left(\exp \left(t v_{i, j}\right)\right)_{k} M$ and $\left(\exp \left(t w_{i, j}\right)\right)_{k} M$ at $t=0$, where $a_{k}:=(1, \ldots, 1, a, 1, \ldots, 1) \in U(N)^{m}$, with $a \in U(N)$ in the $k$-th place. Then by formula (4), we have

$$
\mathrm{d} M=\phi \cdot \frac{1}{2^{N}} \prod_{i} \sqrt{Q^{\prime}\left(x_{i}\right)} \mathrm{d} x_{1} \cdots \mathrm{d} x_{N} \mathrm{~d} \theta_{1} \cdots \mathrm{d} \theta_{N} \mathrm{~d} U,
$$

where

$$
\phi=\left|\wedge_{i<j, k}\left(V_{i, j, k} \wedge W_{i, j, k}\right)\right| .
$$

To calculate $\phi$, let us denote by $B_{i, j, k}, i \neq j$, the derivative of $\left(\exp \left(t E_{i, j}\right)\right)_{k} M$ (note that since $E_{i, j}$ lies only in the complexified Lie algebra of $U(N)^{m}$, we have $\left(\exp \left(t E_{i, j}\right)\right)_{k} M \notin \mathcal{N}_{m}(\lambda, \mathbb{C})$, but this is not important for our considerations). Then equation (5) takes the form

$$
\phi=\left|\wedge_{i \neq j, k} B_{i, j, k}\right| .
$$

Now $\phi$ can be easily calculated. To do so, we note that for a given $i, j$, the transformation $\left(\exp \left(t E_{i, j}\right)\right)_{k}$ changes only the entries $a_{i, j}^{p}$ of $M$. On these entries, it acts by

$$
a_{i, j}^{p} \rightarrow a_{i, j}^{p}+t\left(z_{j}^{p} \delta_{p, k}-z_{i}^{p} \delta_{p, k-1}\right) .
$$


This means that for each $i, j,\left|\wedge_{k} B_{i, j, k}\right|=\left|J_{i, j}\right|$, where

$$
J_{i, j}:=\operatorname{det}\left(\begin{array}{ccccc}
z_{j}^{1} & -z_{i}^{1} & 0 & \cdots & 0 \\
0 & z_{j}^{2} & -z_{i}^{2} & \ddots & \vdots \\
\vdots & 0 & z_{j}^{3} & \ddots & 0 \\
0 & \ddots & 0 & \ddots & -z_{i}^{m} \\
-z_{i}^{m} & 0 & \cdots & 0 & z_{j}^{m}
\end{array}\right)=\prod_{s=1}^{m} z_{j}^{s}-\prod_{s=1}^{m} z_{i}^{s}=z_{j}-z_{i} .
$$

This implies that

$$
\phi=\prod_{i \neq j}\left|J_{i, j}\right|=\prod_{i<j}\left|z_{i}-z_{j}\right|^{2},
$$

as desired.

\subsection{The probability measure with potential function on generalized normal matrices}

Let $W: \mathbb{C} \rightarrow \mathbb{R}$ be a potential function. The probability measure on $\mathcal{N}_{m}(\lambda, D)$ corresponding to this function is defined similarly to the case of usual normal matrices:

$$
P_{N}(M) \mathrm{d} M=Z_{N}^{-1} \exp \left(-N \operatorname{tr} W\left(M_{1} \cdots M_{m}\right)\right) \mathrm{d} M,
$$

$M \in \mathcal{N}_{m}(\lambda, D)$, where $M_{i}$ are the blocks of $M$. Thus in terms of eigenvalues

$$
\begin{aligned}
P_{N}(M) \mathrm{d} M= & \frac{1}{2^{N} Z_{N}} \exp \left\{-N \sum_{j} W\left(z_{j}\right)\right\} \\
& \times \prod_{i} \sqrt{Q^{\prime}\left(x_{i}\right)} \prod_{i<j}\left|z_{i}-z_{j}\right|^{2} \mathrm{~d} x_{1} \cdots \mathrm{d} x_{N} \mathrm{~d} \theta_{1} \cdots \mathrm{d} \theta_{N} \mathrm{~d} U .
\end{aligned}
$$

Example 1. Let us calculate the potential function corresponding to the quadratic potential $\operatorname{Tr}\left(M M^{\dagger}\right)$. We have

$$
\operatorname{Tr}\left(M M^{\dagger}\right)=\sum_{i, j}\left|z_{j}^{i}\right|^{2}=\sum_{i, j}\left(x_{j}+\alpha_{i}\right)=N \sum_{i} \alpha_{i}+m \sum_{j} x_{j} .
$$

Thus if we choose $\alpha_{i}$ so that $\sum_{i} \alpha_{i}=0$ (this can be done in a unique way), then

$$
\operatorname{Tr}\left(M M^{\dagger}\right)=m \sum_{j} x_{j}
$$

so the corresponding potential function is $W(z)=m Q^{-1}\left(|z|^{2}\right)$ (the function $Q$ is invertible on the interval $[-\alpha, \infty)$, where $\left.\alpha=\min \alpha_{i}\right)$.

\section{Equilibrium measure}

\subsection{Some basic facts about equilibrium measure}

Let $D$ be a compact subset of the complex plane $\mathbb{C}$, and $W(z)$ a potential function (a continuous function on $D$ ). Denote by $\mathcal{M}(D)$ the set of the Borel probability measures $\sigma$ on $D$ without point masses, and define the energy of $\sigma$ to be

$$
I_{\sigma}=\int_{D} W(z) \mathrm{d} \sigma(z)+\int_{D} \int_{D} \log |z-w|^{-1} \mathrm{~d} \sigma(z) \mathrm{d} \sigma(w) .
$$


An equilibrium measure for $W$ on $D$ is a measure $\sigma \in \mathcal{M}(D)$ such that

$$
I_{\sigma}=\inf _{\mu \in \mathcal{M}(D)} I_{\mu}
$$

Theorem 3. The equilibrium measure $\sigma$ exists and is unique. It satisfies the equation

$$
W(z)-2 \int_{D} \log |z-w| \mathrm{d} \sigma(w)=C,
$$

where $C$ is a constant, almost everywhere with respect to $\sigma$.

The proof of this theorem can be found in [3].

Note that equation (6) does not have to hold outside the support of $\sigma$.

Note also that if $\sigma$ is absolutely continuous with respect to the Lebesgue measure near a point $z_{0}$ in the interior of $D$, and $\mathrm{d} \sigma=g(z) \mathrm{d}^{2} z$, where $g$ is continuous near $z_{0}$ and $g\left(z_{0}\right)>0$, then $\Delta W=4 \pi g$ near $z_{0}$. This clearly cannot happen at points where $\Delta W \leq 0$. In particular, if $\Delta W \leq 0$ everywhere, then $\mathrm{d} \sigma$ tends to be concentrated on the boundary of $D$.

\subsection{Asymptotic eigenvalue distribution in the normal matrix model}

In Section 2, we defined a measure

$$
P_{N}(M) \mathrm{d} M=J_{N}\left(z_{1}, \ldots, z_{N}\right) \mathrm{d}^{2} z_{1} \cdots \mathrm{d}^{2} z_{N} \mathrm{~d} U
$$

by formula (2). We are interested in the behavior of this measure when $N \rightarrow \infty$. Let $\delta_{z}=$ $\frac{1}{N} \sum_{j=1}^{N} \delta_{z_{j}}$ be the measure on $D$ corresponding to the points $z_{j}$. Then

$$
-\log \left(Z_{N} J_{N}\left(z_{1}, \ldots, z_{N}\right)\right)=N^{2}\left(\int W(\xi) \mathrm{d} \delta_{z}(\xi)+\iint_{\xi \neq \zeta} \log |\xi-\zeta|^{-1} \mathrm{~d} \delta_{z}(\zeta) \mathrm{d} \delta_{z}(\xi)\right) .
$$

This shows that the leading contribution to the integral with respect to the measure $P_{N}(M) \mathrm{d} M$ comes from configurations of eigenvalues $z_{1}, \ldots, z_{N}$ for which the expression in parentheses in the last equation is minimized. This means that in the limit $N \rightarrow \infty$, we should expect the measures $\delta_{z}$ for optimal configurations to converge to the equilibrium measure with potential function $W$.

This indeed turns out to be the case, as shown by the following theorem, proved in [3].

Theorem 4. Let the k-point correlation function be

$$
R_{N}^{(k)}\left(\left(z_{i}\right)_{i=1}^{k}\right)=\int_{D^{N-k}} J_{N}\left(z_{1}, \ldots, z_{N}\right) \prod_{i=k+1}^{N} \mathrm{~d}^{2} z_{i} .
$$

Then the measure

$$
R_{N}^{(k)}\left(\left(z_{i}\right)_{i=1}^{k}\right) \prod_{i=1}^{k} \mathrm{~d}^{2} z_{i}
$$

on $D^{k}$ converges weakly to $\mathrm{d} \sigma^{\otimes k}$, where $\mathrm{d} \sigma$ is the equilibrium measure on $D$, corresponding to the potential function $W$.

In particular, if $k=1$, it means that the eigenvalue distribution tends to the equilibrium measure in $D$ as $N \rightarrow \infty$. 


\subsection{Asymptotic eigenvalue distribution in the generalized normal matrix model}

As we have seen above, the eigenvalue distribution in the generalized normal matrix model is

$$
P_{N}(M) \mathrm{d} M=J_{N, m}\left(z_{1}, \ldots, z_{N}\right) \mathrm{d}^{2} z_{1} \cdots \mathrm{d}^{2} z_{N} \mathrm{~d} U
$$

where

$$
\begin{aligned}
-\log \left(2^{N} Z_{N} J_{N, m}\right)= & N^{2}\left(\int W(\xi) \mathrm{d} \delta_{z}(\xi)+\iint_{\xi \neq \zeta} \log |\xi-\zeta|^{-1} \mathrm{~d} \delta_{z}(\zeta) \mathrm{d} \delta_{z}(\xi)\right) \\
& -\frac{N}{2} \int \log Q^{\prime}\left(Q^{-1}\left(|\xi|^{2}\right)\right) \mathrm{d} \delta_{z}(\xi) .
\end{aligned}
$$

In the limit $N \rightarrow \infty$ the second term becomes unimportant compared to the first one, which implies that Theorem 4 is valid for the generalized normal matrix model. Thus in the limit $N \rightarrow \infty$, the usual and the generalized normal matrix models (with the same potential) are equivalent.

\section{Reconstruction of the boundary of the domain}

In previous sections, we showed that in the normal matrix model and the generalized normal matrix model, when $N \rightarrow \infty$, the eigenvalue distribution converges to an equilibrium measure on $D$ corresponding to some potential function $W$. In this section, we will try to find this measure explicitly in some special cases.

More specifically, we will consider the case when $\Delta W>0$. In this case, if the region $D$ is sufficiently large, it turns out that the equilibrium measure is often absolutely continuous with respect to Lebesgue measure, and equals $\mathrm{d} \sigma=(4 \pi)^{-1} \chi_{E} \Delta W \mathrm{~d}^{2} z$, where $E$ is a region contained in $D$ (the region of eigenvalues), and $\chi_{E}$ is the characteristic function of $E$. More precisely, it follows from Proposition 3.4 in [3] that if there exists a region $E \subset D$ such that $\mathrm{d} \sigma$ satisfies equation (6) in $E$, and the left hand side of this equation is $\geq C$ on $D \backslash E$, then $\mathrm{d} \sigma$ is the equilibrium measure on $D$ for the potential function $W$. Moreover, note that if $E$ works for some $D$ then it works for any smaller $D^{\prime}$ such that $E \subset D^{\prime} \subset D$. So, in a sense, $E$ is independent of $D$. (Here we refer the reader to [5], section 4, where there is a much more detailed and precise treatment of equilibrium measures, without the assumption $\Delta \Phi>0$ ).

Thus let us assume that $E$ exists, and consider the problem of finding it explicitly given the potential $W$.

\subsection{The reconstruction problem}

We will consider the case when $D=D(R)$ is the disk of radius $R$ centered at the origin, and

$$
W(z)=\Phi(z \bar{z})-P(z)-\overline{P(z)}
$$

where $\Phi$ is a function of one variable continuous on $[0, \infty)$ and twice continuously differentiable on $(0, \infty)$, and $P$ a complex polynomial. We assume that $\left(s \Phi^{\prime}(s)\right)^{\prime}$ is positive, integrable near zero, and satisfies the boundary condition $\lim _{s \rightarrow 0} s \Phi^{\prime}(s)=0$. Computing the Laplacian of $W$, we get (taking into account that $\Delta=4 \partial \bar{\partial}$ ):

$$
g(s):=(4 \pi)^{-1} \Delta W=\pi^{-1}\left(\Phi^{\prime}(s)+s \Phi^{\prime \prime}(s)\right),
$$

where $s=z \bar{z}$. Define the measure $\mathrm{d} \sigma=g \mathrm{~d}^{2} z$. 
Suppose that the region $E$ exists, and contains the origin. In this case, differentiating equation (6) with respect to $z$, we have inside $E$ :

$$
\bar{z} \Phi^{\prime}(z \bar{z})-P^{\prime}(z)=\int_{E} \frac{g(w \bar{w})}{z-w} \mathrm{~d}^{2} w .
$$

On the other hand, inside the disk $D$, the function

$$
W_{0}(z):=2 \int_{D} g(w \bar{w}) \log |z-w| \mathrm{d}^{2} w
$$

satisfies the equation $\Delta W_{0}=4 \pi g$, and is rotationally invariant, so

$$
W_{0}(z)=\Phi(z \bar{z})+C^{\prime},
$$

where $C^{\prime}$ is a constant. Hence, differentiating, we get, inside $D$ :

$$
\bar{z} \Phi^{\prime}(z \bar{z})=\int_{D} \frac{g(w \bar{w})}{z-w} \mathrm{~d}^{2} w .
$$

Thus, subtracting (7) from (8), we obtain inside $E$ :

$$
P^{\prime}(z)=\int_{D \backslash E} \frac{g(w \bar{w})}{z-w} \mathrm{~d}^{2} w .
$$

Let $I(s)=\pi \int_{0}^{s} g(t) \mathrm{d} t=s \Phi^{\prime}(s)$. Then $\bar{\partial} I(z \bar{z})=\pi z g(z \bar{z}) \mathrm{d} \bar{z}$. Thus, using Green's formula, we get from (9):

$$
P^{\prime}(z)=\frac{1}{2 \pi i} \int_{\partial D-\partial E} \frac{I(w \bar{w})}{w(z-w)} \mathrm{d} w
$$

where the boundaries are oriented counterclockwise. The integral over the boundary of $D$ is zero by Cauchy's formula, so we are left with the equation

$$
P^{\prime}(z)=\frac{1}{2 \pi i} \int_{\partial E} \frac{I(w \bar{w})}{w(w-z)} \mathrm{d} w .
$$

This equation appeared first in the theory of Hele-Shaw flows on curved surfaces in [4], and it can be solved explicitly by the method of singular points developed in the same paper. Let us recall this method.

\subsection{The singular point method}

Define the Cauchy transform $h_{E}$ of $E$ with respect to the measure d $\sigma$ by

$$
h_{E}(z)=\int_{D \backslash E} \frac{\mathrm{d} \sigma(w)}{z-w}, \quad z \in E .
$$

This is a holomorphic function of $z$ which (as we have just seen) is independent of the radius $R$ of $D$. As we have seen, it is also given by the contour integral

$$
h_{E}(z)=\frac{1}{2 \pi i} \int_{\partial E} \frac{I(w \bar{w})}{w(w-z)} \mathrm{d} w
$$

and in our case we have $h_{E}(z)=P^{\prime}(z)$.

Let $f: D(1) \rightarrow \overline{\mathbb{C}} \backslash E$ be a conformal map, such that $f(0)=\infty$, and $(1 / f)^{\prime}(0)=a \in \mathbb{R}^{+}$ (such a map is unique). 
Lemma 2. The function

$$
\phi(\zeta)=I(f(\zeta) \overline{f(\zeta)})-f(\zeta) h_{E}(f(\zeta))
$$

continues analytically from the unit circle to a holomorphic function outside the unit disk.

Proof. By the Cauchy formula, we have

$$
h_{E}(z)=\frac{1}{2 \pi i} \int_{\partial E} \frac{h_{E}(w)}{w-z} \mathrm{~d} w, \quad \text { for any } z \in E .
$$

So by formula (10), we have

$$
\frac{1}{2 \pi i} \int_{\partial E} \frac{I(w \bar{w}) / w-h_{E}(w)}{w-z} \mathrm{~d} w=0, \quad \text { for any } z \in E .
$$

It follows that the function $I(z \bar{z}) / z-h_{E}(z)$, defined along $\partial E$, can be analytically continued to a holomorphic function outside $E$, which vanishes at infinity. This implies the lemma.

Similarly to [4], this lemma implies the following theorem.

Theorem 5. The function $h_{E}$ is rational if and only if the function $\theta(\zeta)=I(f(\zeta) \overline{f(1 / \bar{\zeta})}$ is. Moreover, the number of poles of $\theta$ is twice of the number of poles of $z h_{E}(z)$. More specifically, if $\zeta_{0}$ and $1 / \bar{\zeta}_{0}$ are poles of $\theta$ of order $m$, then $z_{0}=f\left(\zeta_{0}\right)$ is a pole of order $m$ for $h_{E}(z)$, and vice versa.

Thus, if $h$ is a rational function, then $\theta$ can be determined from $h$ up to finitely many parameters.

After this, $f$ can be reconstructed from $\theta$ using the Cauchy formula. For this, note that the function $I$ is invertible, since $I^{\prime}=g>0$. Also, $\theta$ takes nonnegative real values on the unit circle. Thus, we have

$$
f(\zeta) \overline{f(1 / \bar{\zeta})}=I^{-1}(\theta(\zeta))
$$

Taking the logarithm of both sides, we obtain

$$
\log (\zeta f(\zeta))+\log \left(\zeta^{-1} \overline{f(1 / \bar{\zeta})}\right)=\log I^{-1}(\theta(\zeta)) .
$$

Thus we have

$$
\begin{aligned}
& f(\zeta)=a \zeta^{-1} \exp \left(\frac{1}{2 \pi i} \int_{|u|=1} \frac{\log I^{-1}(\theta(u))}{u-\zeta} \mathrm{d} u\right), \\
& a=\exp \left(-\frac{1}{4 \pi i} \int_{|u|=1} \frac{\log I^{-1}(\theta(u))}{u} \mathrm{~d} u\right) .
\end{aligned}
$$

The unknown parameters of $\theta$ can now be determined from the cancellation of poles in Theorem 5, similarly to the procedure described in [10]. We note that the knowledge of the function $h_{E}$ is not sufficient to determine $E$ (for example if $E$ is a disk of any radius centered at the origin then $\left.h_{E}=0\right)$. To determine the parameters completely, we must also use the information on the area of $E$ :

$$
\int_{E} \mathrm{~d} \sigma=-\frac{1}{2 \pi i} \int_{|u|=1} \frac{\theta(u)}{f(u)} f^{\prime}(u) \mathrm{d} u=1 .
$$




\subsection{The polynomial case}

In particular, in our case,

$$
h_{E}(z)=P^{\prime}(z)=a_{1}+a_{2} z+\cdots+a_{d} z^{d-1},
$$

which implies that $\theta(\zeta)=\sum_{j=-d}^{d} b_{j} \zeta^{j}$, and $\bar{b}_{j}=b_{-j}$.

So we get

$$
\begin{aligned}
& f(\zeta)=a \zeta^{-1} \exp \left(\frac{1}{2 \pi i} \int_{|u|=1} \frac{\log I^{-1}\left(\sum_{j=-d}^{d} b_{j} u^{j}\right)}{u-\zeta} \mathrm{d} u\right), \\
& a=\exp \left(-\frac{1}{4 \pi i} \int_{|u|=1} \frac{\log I^{-1}\left(\sum_{j=-d}^{d} b_{j} u^{j}\right)}{u} \mathrm{~d} u\right) .
\end{aligned}
$$

Finally, note that if the coefficients of the polynomial $P$ are small enough, then all our assumptions are satisfied: the region $E$ exists (in fact, it is close to a disk), and contains the origin. Also, in this case the left hand side of equation (6) is $\geq C$, which implies that the equilibrium measure in this case (and hence, the asymptotic eigenvalue distribution) is the measure $\mathrm{d} \sigma$ in the region $E$.

Example 2. Consider Example 1: the generalized normal matrix model with the density $\exp \left(-\beta \operatorname{tr}\left(M M^{\dagger}-P(M)-P(M)^{\dagger}\right)\right.$. As we showed, in this case $\Phi(s)=m \beta Q^{-1}(s)$. So a short computation shows that

$$
\pi m^{-1} \beta^{-1} g(Q(x))=\frac{Q(x)^{2}}{Q^{\prime}(x)^{3}} \sum_{i} \frac{1}{\left(x+\alpha_{i}\right)^{2}} .
$$

This implies that $g>0$, i.e. our analysis applies in this case.

\subsection{Some explicit solutions}

Consider the case $\Phi(s)=C s^{b}, C, b>0$. For example, in the generalized normal matrix model with $\alpha_{i}=0$ and potential term as in Example 1, one has $\Phi(s)=m s^{1 / m}$, which is a special case of the above.

We have $g(s)=\pi^{-1} C b^{2} s^{b-1}$, so our analysis applies (note that if $b<1$ then $g$ is singular at zero, but the singularity is integrable and thus nothing really changes in our considerations), and $I(s)=C b s^{b}$. Thus the integral in (11) can be computed explicitly (by factoring $\theta$ ), and the formula for the conformal map $f$ simplifies as follows:

$$
f(\zeta)=(a \zeta)^{-1} \prod_{j=1}^{d}\left(1-\zeta \zeta_{j}^{-1}\right)^{1 / b} .
$$

The parameters $a>0$ and $\zeta_{j}$ are determined from the singularity conditions and the area condition.

Consider for simplicity the example $d=1$. In this case we have

$$
h_{E}(z)=K,
$$


and we can assume without loss of generality that $K \in \mathbb{R}$. Then

$$
f(\zeta)=(a \zeta)^{-1}(1+\beta \zeta)^{1 / b}, \quad \beta \in \mathbb{R},
$$

and

$$
\theta(\zeta)=C b a^{-2 b}(1+\beta \zeta)\left(1+\beta \zeta^{-1}\right) .
$$

The residue of $\theta$ at zero is thus $C b \beta a^{-2 b}$. Thus the singularity condition says

$$
C b \beta a^{1-2 b}=K \text {. }
$$

The area condition is

$$
1=C b a^{-2 b}\left(1+\beta^{2}\left(1-b^{-1}\right)\right) .
$$

Thus we find $\beta=K C^{-1} b^{-1} a^{2 b-1}$, and the equation for $a$ has the form

$$
C b a^{-2 b}+C^{-1} b^{-1} K^{2} a^{2 b-2}\left(1-b^{-1}\right)=1 .
$$

Remark 2. This example shows that to explicitly solve the generalized (as opposed to the usual) normal matrix model in the $N \rightarrow \infty$ limit with the quadratic (Gaussian) potential, one really needs the technique explained in Section 5 of this paper, and the techniques of [3] are not sufficient.

\section{Acknowledgements}

P.E. is grateful to G. Felder and P. Wiegmann for useful discussions. The work of P.E. was partially supported by the NSF grant DMS-0504847.

\section{References}

[1] Chau L.-L., Zaboronsky O., On the structure of correlation functions in the normal matrix model, Comm. Math. Phys. 196 (1998), 203-247, hep-th/9711091.

[2] Crawley-Boevey W., Holland M.P., Noncommutative deformations of Kleinian singularities, Duke Math. J. 92 (1998), 605-635.

[3] Elbau P., Felder G., Density of eigenvalues of random normal matrices, Comm. Math. Phys. 259 (2005), 433-450, math.QA/0406604.

[4] Entov V.M., Etingof P.I., Viscous flows with time-dependent free boundaries in a non-planar Hele-Shaw cell, Euro. J. Appl. Math. 8 (1997), 23-35.

[5] Hedenmalm H., Makarov N., Quantum Hele-Shaw flow, math.PR/0411437.

[6] Kostov I.K., Krichever I., Mineev-Weinstein M., Wiegmann P.B., Zabrodin A., The $\tau$-function for analytic curves, in Random Matrix Models and Their Applications, Math. Sci. Res. Inst. Publ., Vol. 40, Cambridge Univ. Press, Cambridge, 2001, 285-299.

[7] Krichever I., Marshakov A., Zabrodin A., Integrable structure of the Dirichlet boundary problem in multiplyconnected domains, Comm. Math. Phys. 259 (2005), 1-44, hep-th/0309010.

[8] Marshakov A., Wiegmann P.B., Zabrodin A., Integrable structure of the Dirichlet boundary problem in two dimensions, Comm. Math. Phys. 227 (2002), 131-153, hep-th/0109048.

[9] Oas G., Universal cubic eigenvalue repulsion for random normal matrices, Phys. Rev. E 55 (1997), 205-211, cond-mat/9610073.

[10] Varchenko A.N., Etingof P.I., Why the boundary of a round drop becomes a curve of order four, AMS, Providence, 1992.

[11] Wiegmann P.B., Zabrodin A., Conformal maps and integrable hierarchies, Comm. Math. Phys. 213 (2000), 523-538, hep-th/9909147.

[12] Wiegmann P.B., Zabrodin A., Large scale correlations in normal non-Hermitian matrix ensembles, J. Phys. A: Math. Gen. 36 (2003), 3411-3424, hep-th/0210159. 